\title{
Genome Analysis of F. nucleatum sub spp vincentii and Its Comparison With the Genome of $F$. nucleatum ATCC 25586
}

\author{
Vinayak Kapatral, ${ }^{1}$ Natalia Ivanova, lain Anderson, Gary Reznik, \\ Anamitra Bhattacharyya, Warren L. Gardner, Natalia Mikhailova, Alla Lapidus, \\ Niels Larsen, Mark D'Souza, Theresa Walunas, Robert Haselkorn, Ross Overbeek, \\ and Nikos Kyrpides
}

Integrated Genomics Inc., Chicago, Illinois 60612, USA

\begin{abstract}
We present the draft genome sequence and its analysis for Fusobacterium nucleatum sub spp. vincentii (FNV), and compare that genome with $F$. nucleatum ATCC 25586 (FN). A total of $441 \mathrm{FNV}$ open reading frames (ORFs) with no orthologs in $\mathrm{FN}$ have been identified. Of these, 118 ORFs have no known function and are unique to FNV, whereas 323 ORFs have functional orthologs in other organisms. In addition to the excretion of butyrate, $\mathrm{H}_{2} \mathrm{~S}$ and ammonia-like FN, FNV has the additional capability to excrete lactate and aminobutyrate. Unlike FN, FNV is likely to incorporate galactopyranose, galacturonate, and sialic acid into its O-antigen. It appears to transport ferrous iron by an anaerobic ferrous transporter. Genes for eukaryotic type serine/threonine kinase and phosphatase, transpeptidase E-transglycosylase PbplA are found in FNV but not in FN. Unique $A B C$ transporters, cryptic phages, and three types of restriction-modification systems have been identified in FNV. ORFs for ethanolamine utilization, thermostable carboxypeptidase, $\gamma$ glutamyl-transpeptidase, and deblocking aminopeptidases are absent from FNV. FNV, like FN, lacks the classical catalase-peroxidase system, but thioredoxin/glutaredoxin enzymes might alleviate oxidative stress. Genes for resistance to antibiotics such as acriflavin, bacitracin, bleomycin, daunorubicin, florfenicol, and other general multidrug resistance are present. These capabilities allow Fusobacteria to survive in a mixed culture in the mouth.
\end{abstract}

[The sequence of Fusobacterium nucleatum sub spp vincentii is deposited in GenBank with the accession no. AABFO1000000.]

Nearly $15 \%-20 \%$ of the human population suffers from periodontal diseases that result in gum decay and tooth loss. Among the 500 bacterial species that exist in the mouth, $F u$ sobacterium nucleatum is the dominant species. F. nucleatum occurs in lower numbers at a healthy site in the mouth, but significantly higher at periodontal disease sites (Moore and Moore 1994). It serves as a bridge between the early colonizers such as Streptococcus gordonii, S. oralis, S. mitis, Actinomyces, Capnocytophaga spp., Propionibacterium, Veillonella spp., etc., and the late colonizers including Porphorymonas gingivalis, Bacteroidies forsythus, Actinobacillus actinomycetemcomitans, Eubacterium spp., and Campylobacter spp. F. nucleatum is one of the primary bacteria responsible for tooth and gum decay and bad mouth odor (Bolstad et al. 1996; Kolenbrander et al. 2002). It also produces tissue irritants that inhibit fibroblast cell division and wound healing processes. Unlike $P$. gingivalis, Fusobacteria induces the expression of $\beta$-defensin 2 from the epithelial cells (Krishnaprakornkit et al. 2000). Species belonging to the Fusobacterium genus also cause other infections such as Lemierre's syndrome, tropical skin ulcers, infection of the heart, joints, liver, and spleen (Bolstad et al. 1996). Fusobacteria are a heterogeneous group of Gram-negative bacteria that are classified into four known subspecies, on the basis of

'Corresponding author.

E-MAIL vinayak@integratedgenomics.com; FAX (312) 226-9446. Article and publication are at http://www.genome.org/cgi/doi/10.1101/ gr.566003. differences in the electrophoretic patterns of whole-cell proteins, DNA methylation, DNA homology, and glutamate dehydrogenase (Gharbia and Shah 1988, 1990, 1992; Dzink et al. 1990; Bolstad and Jensen 1993; Bolstad et al. 1996). These subspecies are viz., vincentii, polymorphum, fusiforme and animalis, of which only the subspecies vincentii is associated with periodontal disease (Dzink et al. 1990; Gharbia and Shah 1992). These subspecies vary in their ability to attach and invade human gingival epithelial cells as well as in stimulating the production of pro-inflammatory interleukin-8 (IL-8) (Han et al. 2000).

We have recently analyzed the genome of $F$. nucleatum strain ATCC 25586 (FN) (Kapatral et al. 2002) and have elucidated its metabolic and pathogenic capabilities. Here, we present the draft sequence and analysis of a second subspecies, F. nucleatum sub spp vincentii ATCC 49256 (FNV) that was also isolated from a human periodontal surface (Dzink et al. 1990). We also have compared the genome features of the sequenced region of FNV with that of $F$. nucleatum (Kapatral et al. 2002) and have identified their unique and common characteristics.

\section{RESULTS}

Genome Sequence of $F$. nucleatum sub spp vincentii

A draft genome sequence of FNV with $\sim 6.4 \times$ coverage was generated. The FNV genome sequence covers about $98 \%$ of 
the FN genome (Kapatral et al. 2002). A total of 2277 open reading frames (ORFs) were identified in FNV, of which 1576 (69\%) were assigned a putative function (Table 1). Of the remaining 701 ORFs (31\%), 570 are hypothetical proteins, and 118 ORFs (5\%) are unique to FN. A total of $1326(57 \%)$ of the ORFs belong to orthologous clusters and 453 ORFs (20\%) to paralog clusters.

\section{Global Comparison}

A total of 2088 protein families (from a total of 4324 ORFs) from FN (completed genome) and FNV (unfinished genome) were identified using the clustering software Workbench with a threshold E-value of $10^{-10}$ (Table 2). Of these, 1339 clusters (comprising 3537 ORFs) were found to be common to both genomes. Each of these clusters has at least one ORF from either of the two genomes. A total of 329 clusters (comprising 346 ORFs) that are absent in FNV have been identified in FN, of which only 70 ORFs are unique. Similarly, 420 clusters (comprising 441 ORFs) that are absent from FN have been identified in FNV, of which 118 ORFs are unique and show no similarity to ORFs in the genomes in ERGO. Of the 323 ORFs, 130 have predicted function and are absent in FN (Table 3).

Gene context comparison further revealed a close relationship between the two genomes. The number of ORFs in chromosomal clusters was found to be 763 (33\%) in FNV and $825(40 \%)$ in FN, suggesting that the chromosomal order of about one-third of all ORFs from the two genomes is conserved with at least one more organism in the ERGO database with a phylogenetic distance larger than 0.1 (Overbeek et al. 1999; Table 4). Comparing the two F. nucleatum genomes, we identified 1230 ORFs from each of the two genomes (60\% of $\mathrm{FN}$ and $54 \%$ of $\mathrm{FNV}$ ) forming 258 common chromosomal clusters.

Comparative functional roles of the two F. nucleatum genomes are given in Figure 1. A functional role is defined as a single step in a pathway, and one role may participate in more than one pathway. In carbohydrate, coenzyme, cofactor, and nucleic acid metabolism subsystems, the number of functional roles is higher in FNV than FN. In general, Fusobacteria devote relatively fewer genes to subsystems such as secretion, virulence, bioenergetics, amino acid biosynthesis, one carbon metabolism, and signal transduction, compared with most other bacteria. We conclude that FN and FNV share several common metabolic capabilities that allow them to occupy a similar periodontal niche (Dzink et al. 1990).

\begin{tabular}{lc}
$\begin{array}{l}\text { Table 1. Genome Statistics of } F \text {. nucleatum sub spp } \\
\text { vincentii (FNV) }\end{array}$ \\
\hline Features & ATCC-49256 \\
\hline Total DNA bases & $2,118,259$ \\
DNA Contigs & 302 \\
Fold coverage & -6.4 \\
ORF number & 2,277 \\
ORFs with assigned function & $1,576(69)$ \\
ORFs without assigned function & $701(31)$ \\
ORFs without function or similarity & $117(5)$ \\
ORFs without function with similarity & $584(25)$ \\
ORFs in ortholog clusters & $1,317(58)$ \\
ORFs in paralog clusters & $453(20)$ \\
\hline
\end{tabular}

Numbers in brackets represent percentage of ORFs.

\begin{tabular}{|c|c|c|c|c|}
\hline Genomes & $\begin{array}{l}\text { Number of } \\
\text { clusters }\end{array}$ & $\begin{array}{l}\text { ORFS in } \\
\text { clusters }\end{array}$ & FN ORFs & FNV ORFs \\
\hline$F N+F N V$ & 1339 & 3537 & 1701 & 1836 \\
\hline $\mathrm{FN}$ & 329 & 346 & 346 & - \\
\hline FNV & 420 & 441 & - & 441 \\
\hline Total & 2088 & 4324 & 2047 & 2277 \\
\hline
\end{tabular}

\section{Ribosomal RNA, Transfer RNA, and Protein Synthesis}

The number, types, and organization of the RNA species in FNV are similar to that in FN (Kapatral et al. 2002). One of the $r r n$ operons has the ORFs for tRNA-ala, tRNA-Ile located between the 16S and 5S RNA. A total of 20 tRNA species for all of the amino acids have been identified.

Genes for large ribosomal protein subunits L25P, L34P, and L36P, and the small ribosomal protein subunits S21P, $\mathrm{S} 22 \mathrm{P}$, and S31P are absent both in the sequenced regions of the FNV and FN (Kapatral et al. 2002). It therefore appears that these subunits are not necessary for protein translation in Fusobacteria. The gene for the tRNA ligase for glutamine is absent in FNV, as in FN (Kapatral et al. 2002), glutamine is probably synthesized on a tRNA by the transfer of an amide group to the charged glutamyl (Gln)-tRNA by the glu-tRNA amidotransferase.

\section{Repair, Replication, and Cell Division}

An unusual DNA photolyase protein involved in the direct repair of UV-induced damage is present in both Fusobacterium sub spp. The photolyase activity resides in a conserved fusion protein, with the carboxy-terminal domain identical to the spore photolyase of Bacillus spp. Both Fusobacterium sub spp. have genes to repair alkylated DNA base damage. Orthologs of Escherichia coli genes ada, alkA, alkB, mutM, and tag that are involved in repair of alkylation damage are absent, suggesting that exposure to alkylating agents is minimal in the oral ecological niche. However, genes involved in repair of oxidatively damaged bases (e.g., mutT, mutY, ung) are present in both subspecies.

The ORFs encoding the cell division proteins, except for FtsX protein, are present in FNV. FtsX is an inner membrane protein and is essential for cell division in E. coli, however, its role in Fusobacteria is not known. The ftsAZ operon is downstream of the ORF for D-alanine-D-alanine ligase in FNV, as in most Gram-negative bacteria. Genes for cell division inhibition and the glucose-inhibited division (gidAB operon) are identical in both genomes. However, FNV has two additional paralogs of the gidA gene. Two copies of the cell shapedetermining proteins RodA, MreB, and MreC have been identified in both genomes and are similar to those found in $E$. coli and Bacillus subtilis. Neither of the Fusobacteria genomes has the rod-shape determination MreD gene.

\section{DNA Modification}

Unlike FN, FNV has several types of restriction-modification systems (RM) such as type I, type II, type III, and 5-methylcytosine-specific (mrr) restriction systems (Table 5). There is one complete set for type-I restriction with methylase subunit (FNV01877), modification subunit (FNV01876), and the re- 
Table 3. Functions in $F$. nucleatum sub spp vincentii That Are Not Found in F. nucleatum (FN) but Have Orthologs in Other Genomes

1) DNA related

1. DNA replication protein DnaC

2. Chaperone protein DnaK (2 ORFs)

3. RecT protein

4. Replication initiator protein

5. Restriction enzyme $B c q l \alpha$ subunit, $\beta$ subunit (EC 3.1.21-)

6. Type I restriction-modification system methyltransferase subunit

7. Type I restriction-modification system restriction subunit (EC 3.1.21.3)

8. Type I restriction-modification system specificity subunit

9. Type II restriction-modification system restriction subunit

10. Methylcytosine-specific restriction enzyme MRR (EC 3.1.21.-)

11. Integrase/recombinase

2) Transporters

1. Dipeptide transport ATP-binding protein dppD

2. Efflux pump

3. Ferrous iron transport protein $A$, Ferrous iron transport protein B

4. Transporter, unknown

3) Metabolic enzymes

1. Sugar epimerase/dehydratase

2. Ribosomal-protein-alanine acetyltransferase (EC 2.3.1.128)

3. UDP-galactopyranose mutase (EC 5.4.99.9)

4. UDP-glucose 6-dehydrogenase (EC 1.1.1.22)

5. VI polysaccharide biosynthesis protein VIPC/TVIE

6. Ribosomal-protein-alanine acetyltransferase (EC 2.3.1.128)

7. Anaerobic sulfite reductase subunit $A$, subunit $B$, subunit C (EC 1.8.1 -)

8. Cytidylate kinase (EC 2.7.4.14)

9. Glycosyl transferase (EC 2.4.-.-) (ORFs)

10. Glutamine synthetase (EC 6.3.1.2)

11. Glycosyltransferase involved in cell wall biogenesis (EC 2.4. -. - )

12. Endo- 1 , 4- $\beta$-xylanase (EC 3.2.1.8)

13. L-ribulose-5-phosphate 4-epimerase (EC 5.1.3.4)

14. Nucleotide sugar synthetase

15. Putative hydrolase of the HD superfamily

16. Acylneuraminate cytidylyltransferase (NeuA)

17. N-acetylgucosamine-6-phosphate 2-epimerase (NeuC)

18. UDP-galactoyranose mutase

19. UDP-glucose 6-dehydrogenase

20. UDP-glucuronate 4-epimerase

21. Glucose-1-phosphate cytidylyltransferase

22. CDP-glucose 4,6-dehydratase

23. CDP-4-ketO6-deoxyglucose dehydratase

4) Phage proteins

1. Phage regulatory proteins (2 ORFs)

2. Phage PBSX proteins (6 ORFs)

3. Phage structural proteins ( $8 \mathrm{ORFs}$ )

4. Phage proteins (7 ORFs)

5. Unknown phage proteins ( $87 \mathrm{ORFs}$ )

5) Others

1. Membrane protein LAPB

2. NifU-like protein

3. ATP/GTP-binding protein

4. Competence-damage protein $\mathrm{Cin} A$

5. Gene D protein

6. Sensor protein FixL (EC 2.7.3.-)

7. Terminase large subunit

8. RhuM protein

6) Hypotheticals

1. Hypothetical cytosolic protein (2 ORFs)

2. Hypothetical membrane spanning protein (2 ORFs)

3. Hypothetical protein (6 ORFs) striction subunit (RFNV01875). There are other orphan ORFs with methylase (FNV00414 and RFNV00415), modification (FNV00161), and restriction (FNV00634 and FNV00635) subunits; these might as well be independent systems. The amino-acid sequence of the R subunit of the type-II RM system from FNV is similar to that of the DpnII-type restriction enzyme of Streptococcus pneumoniae. Interestingly, the type-II $\mathrm{R}$ subunit is clustered on the chromosome with a 5-methylcytosine-specific mrr endonuclease (FNV01634) in FNV. The type-III RM system is similar to that found in $N$. meningitidis serotype C (strain FAM18). In addition, FNV has two closely clustered type-III modification subunits with predicted $\mathrm{N}^{6}$ specific adenine methylase activity.

\section{Metabolism}

Fusobacteria obtain energy from sugar and amino acid fermentation. The preferred substrates are amino acids such as glutamine, glutamate, histidine, lysine, and serine. The pathway for glutamate fermentation to butyrate is identical in both strains. The product, 2-butenoyl-CoA, can be converted to butyrate and the ORFs for a butyrate fermentation pathway are similar to those of FN (Kapatral et al. 2002). Although the ORF with predicted glutamate mutase activity (methylaspartate mutase) is found in both strains, the ORF for the enzyme in the subsequent step (methylaspartate ammonia-lyase) of the mesaconate pathway is absent in both $\mathrm{FN}$ and sequenced regions of FNV. Therefore, the role of glutamate mutase is not clear in F. nucleatum. Genes for the 4-aminobutyrate pathway of glutamate fermentation are absent from both FN and FNV (Fig. 2).

The hut operon (histidine utilization) is similar to that present in FN, but the histidine degradation pathway is similar to that of $B$. subtilis. Both FN and FNV can degrade either L-lysine or D-lysine, like Clostridium spp and $P$. putida spp oleovorans. The operon encoding enzymes for lysine utilization is similar to that of $P$. gingivalis, including lysine permease, lysine 2,3-aminomutase, and a bifunctional D-lysine 5,6aminomutase gene, like that of Clostridium sticklandii (Baker et al. 1973; Chang and Frey 2000).

Enzymes for serine degradation, including L-serine dehydratase, are found in both species. D-serine dehydratase, together with a putative D-serine permease was found only in FN. Other amino acids, including arginine, cysteine, and threonine can also be degraded by both species. ORFs for methionine $\gamma$-lyase, found in both genomes, are homologous to methionine $\gamma$-lyase from Treponema denticola and T. vaginalis. Methionine lyase is involved in the production of volatile sulfur compounds, such as methanethiol, from methionine and cysteine. Both FN and FNV are capable of degrading Dand L- threonine due to the presence of ORFs for threonine aldolase and threonine dehydratases. One of the products of

Table 4. ORFs in Chromosomal Clusters (Potential Operons) of $F$. nucleatum (FN) and $F$. nucleatum sub spp vencentii (FNV)

\begin{tabular}{lccc}
\hline Species & $\begin{array}{c}\text { Number } \\
\text { of ORFs }\end{array}$ & $\begin{array}{c}\text { ORFs in clusters } \\
(\text { distance }>\mathbf{0 . 1})\end{array}$ & $\begin{array}{c}\text { Percent in } \\
\text { clusters }\end{array}$ \\
\hline FN & 2067 & 825 & 40 \\
FNV & 2297 & 763 & 33 \\
\hline
\end{tabular}

(Overbeek et al. 1999)

\section{Genome Research}




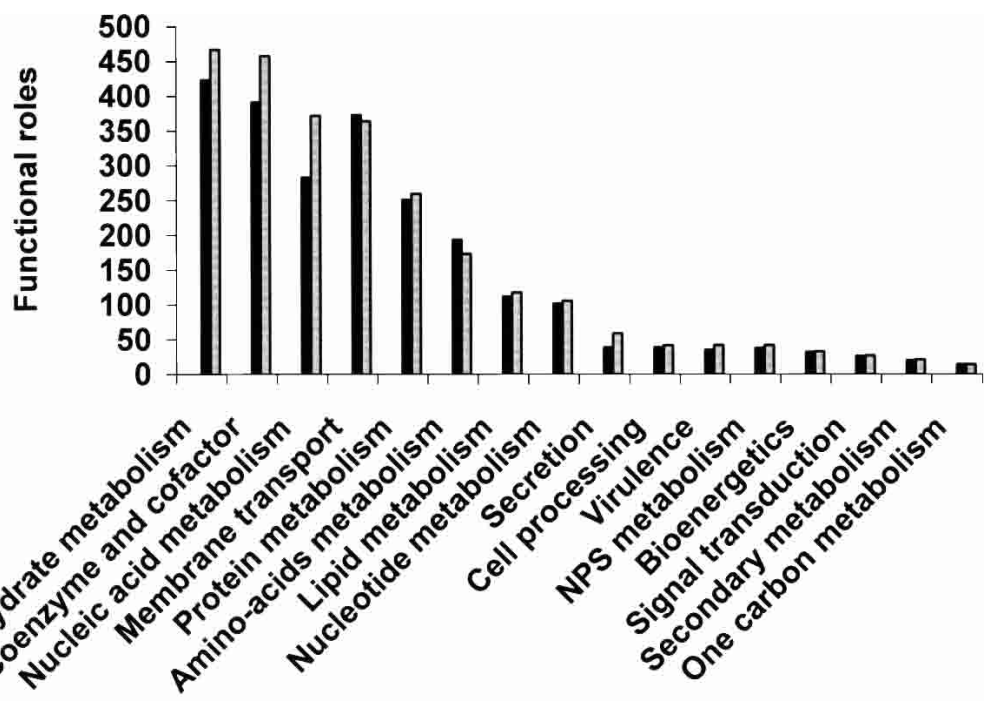

Figure 1 Comparative functional roles in the two species F. nucleatum ATCC 25586 (black) and F. nucleatum sub spp vincentii (gray). A functional role is defined as a step in a pathway. NPS refers to nitrogen, phosphorus, and sulfur metabolism.

methionine and threonine degradation is 2-oxobutanoate, which is usually converted to propanoyl-CoA by the pyruvate dehydrogenase complex. However, the ORF for pyruvate dehydrogenase (lipoamide dependent) is not found in FN or FNV. Instead, an ORF similar to the Clostridium spp. pyruvateflavodoxin (or ferredoxin) oxidoreductase is present. Propionate and 2-hydroxybutyrate were shown to be the major products of D- or L-threonine and 2-oxobutanoate fermentation in Fusobacterium spp. (Carlier et al. 1997). Pyruvate (flavodoxin-dependent) oxidoreductase converts 2-oxobutanoate to propionate, alternatively, D- and L-lactate dehydrogenases can reduce 2-oxobutanoate to 2-hydroxybutyrate. In any event, the end products of these pathways contribute to mouth odor.

The eut operons (eutSPQTDMNEG, eutHABCLK) for ethanolamine utilization are absent in sequenced regions of FNV, whereas these operons are present in FN (Kapatral et al. 2002). The loss is evident as the flanking ORFs dihydropteroate synthase and phosphoserine phosphatase are present in FNV (Fig.

Table 5. Comparison of the Restriction-Modification Systems in F. nucleatum sub spp.

\begin{tabular}{|c|c|c|c|c|c|}
\hline \multirow{2}{*}{$\begin{array}{l}\begin{array}{l}\text { Restriction } \\
\text { modification }\end{array} \\
\text { Type } 1\end{array}$} & \multicolumn{2}{|c|}{$\begin{array}{l}\text { F. nucleatum } \\
\text { ATCC25585 }\end{array}$} & \multicolumn{3}{|c|}{ F. nucleatum spp vincentii } \\
\hline & \multicolumn{2}{|c|}{ ND } & \begin{tabular}{l}
\multicolumn{1}{c}{$R$} \\
FNV01875 \\
FNV00634 \\
FNV00635
\end{tabular} & $\begin{array}{c}M \\
\text { FNV01877 } \\
\text { FNV00161 }\end{array}$ & $\begin{array}{c}\stackrel{S}{\text { FNV01876 }} \\
\text { FNV00414 } \\
\text { FNV00415 }\end{array}$ \\
\hline Type II & \multicolumn{2}{|c|}{ ND } & $\begin{array}{c}R \\
\text { FNV01635 }\end{array}$ & \multicolumn{2}{|c|}{$\begin{array}{c}M \\
\text { ND }\end{array}$} \\
\hline Type II & $\begin{array}{c}R \\
\text { FN01112 }\end{array}$ & $\begin{array}{c}M \\
\text { FN01113 }\end{array}$ & $\begin{array}{c}R \\
\text { FNV02230 }\end{array}$ & \multicolumn{2}{|c|}{$\begin{array}{c}M \\
\text { FNV02229 } \\
\text { FNV02225 }\end{array}$} \\
\hline 5-meC-specific & \multicolumn{2}{|c|}{ ND } & \multicolumn{3}{|c|}{ FNV01634 (mrr) } \\
\hline
\end{tabular}

Plus sign denotes a frameshift.

aTruncated gene.

${ }^{\mathrm{b}} \mathrm{A}$ gene duplication.

(ND) Not detected. absent.
3). Both the Fusobacterium sub spp. can utilize glucose, galactose, fructose, citrate, and glycerol and have genes for sialic acid and N-acetylglucosamine utilization. FNV has ORFs for malolactic permease and malolactic enzyme-like S. mutans (Fig. 4). Malate permease is used for uptake of malate and export of lactate, thus allowing growth of FNV in medium-containing malate. These ORFs are absent in FN. Genes for anaerobic succinate degradation (4hydroxybutyrate dehydrogenase, 4-hydroxybutyryl-CoA dehydratase, and 4-hydroxybutyrate-CoA transferase), similar to those found in C. kluyveri are found in FNV, but not in FN. The degraded product, crotonyl-CoA, feeds into butyrate fermentation. Genes for glucose, galactose, and fructose utilization (EmbdenMeyerhoff pathway, Leloir pathway, and fructose-specific PTS/1-phosphofructokinase) are conserved in both the genomes. All three types of sugars are converted to pyruvate and then to fermentation products such as formate, lactate, and butyrate or used for biosynthesis. Amino acid and fatty acid precursors, oxaloacetate, and acetyl-CoA, are produced by phosphoenolpyruvate carboxykinase and pyruvate (flavodoxin dependent) oxidoreductase, respectively. In both of the Fusobacterium sub spp., ORFs for the TCA cycle enzymes and transaldolases are

\section{Lipopolysaccharide}

Despite the close relatedness between the two subspecies, the O-antigenic polysaccharide composition is strikingly different. Although there is no biochemical evidence, FNV likely incorporates sialic acid in its $\mathrm{O}$-antigen as it contains the ORFs for acylneuraminate cytidylyltransferase (NeuA) and N-acetylglucosamine-6-phosphate 2-epimerase (NeuC), these genes are absent in FN (Kapatral et al. 2002). Further, ORFs for UDPgalactopyranose mutase, UDP-glucose 6-dehydrogenase, and UDP-glucuronate 4-epimerase have been identified in FNV, suggesting that the $\mathrm{O}$-antigen might also contain galactopyranose and galacturonate. FNV also has the ORFs for glucose1-phosphate cytidylyltransferase, CDP-glucose 4,6-dehydratase, and CDP-4-keto 6-deoxyglucose dehydratase, which participate in synthesis of 3, 6-dideoxyhexoses, such as ascarylose, paratose, tyvelose, and abequose. However, the fourth ORF in this cluster has the sugar epimerase/dehydratase domain and is homologous to the $\mathrm{WbdJ}$ protein of the O-antigen cluster of E. coli O111 (Wang et al. 1998). The Oantigen of $E$. coli $\mathrm{O} 111$ contains colitose (3,6-dideoxysugar), and $\mathrm{WbdJ}$ catalyzes the epimerization and reduction reaction in the synthesis of GDP-colitose (Wang et al. 1998). Thus, we infer that the WbdJ ortho$\log$ of FNV could catalyze similar epimerization and reduction of 


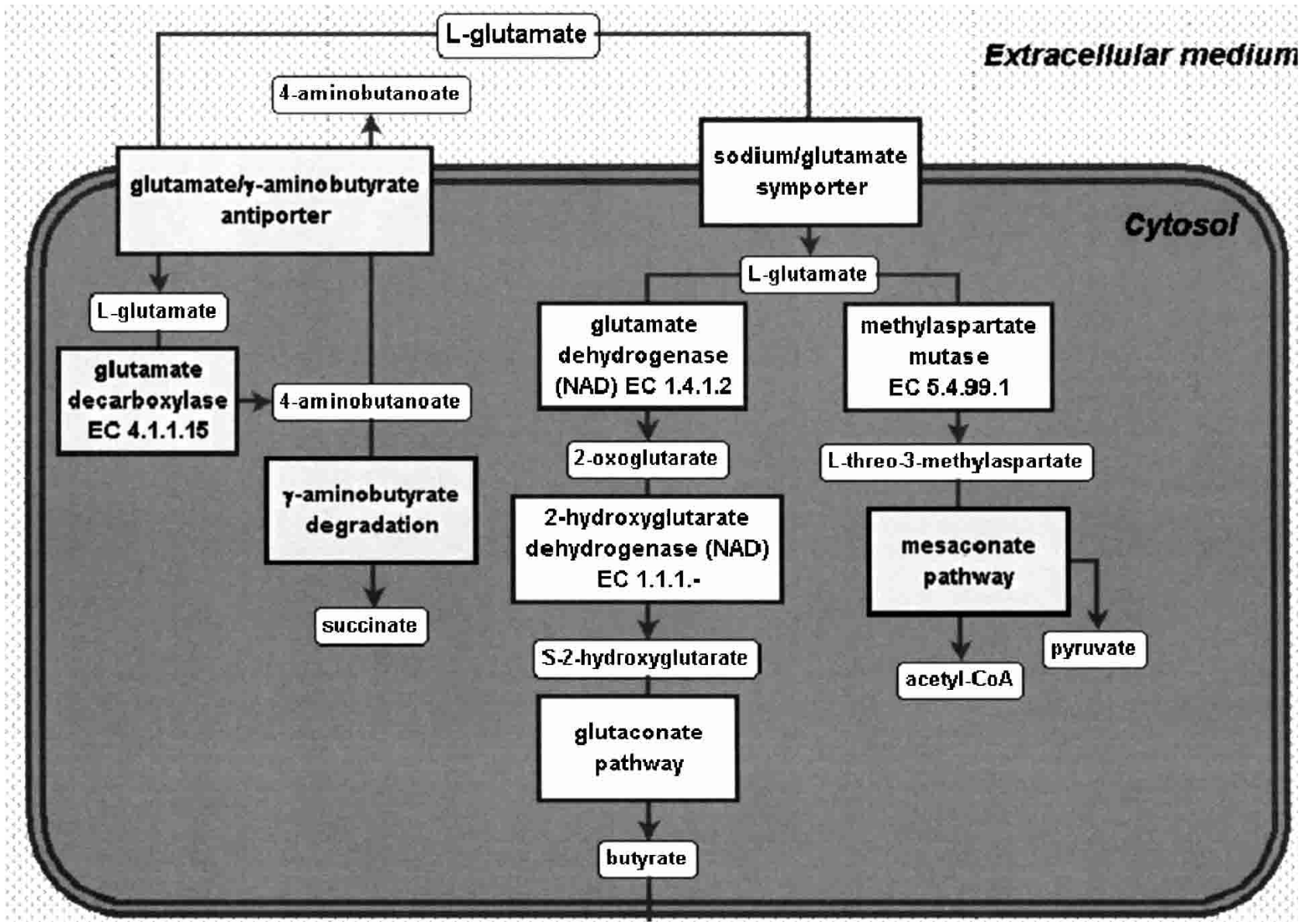

Figure 2 Analysis of glutamate and butyrate pathways in Fusobacteria. White blocks represent the enzymes/pathways present in both FN and FNV. Gray block represents the absence of a pathway in both genomes.

CDP-3, 6-dideoxy-L-glycero-D-glycero-4-hexulose to produce CDP-ascarylose. In contrast, FN has an ORF with homology to a bifunctional UDP-N-acetylglucosamine $\mathrm{C}_{6}$ dehydratase $/ \mathrm{C}_{4}$ reductase, similar to the $\mathrm{WbpM}$ protein of $P$. aeruginosa O6 (Belanger et al. 1999) and to the FlaA1 protein of Helicobacter pylori (Creuzenet et al. 2000) that is involved in biosynthesis of 6-deoxyaminohexose UDP-N-acetylquinovosamine. However, analysis of chromosomal clusters in FN suggests that it produces different deoxyaminosugars, such as bacillosamine in F. necrophorum (Hermansson et al. 1993) and quinovosamine in F. nucleatum JCM 8352 (Onoue et al. 1996).
Amino Acid Biosynthesis

In general, Fusobacteria import amino acids from the external milieu. Amino-acid synthesis in FNV is similar to that of FN (Kapatral et al. 2002). Neither of the Fusobacterium sub spp. have genes for diaminopimelate and lysine biosynthesis, however, the peptidoglycan of Fusobacteria contain lanthionine instead of 2,6-diaminopimelate; the synthesis of lanthionine in Fusobacteria is not known. (Kapatral et al. 2002).

\section{Peptidases}

Because several essential amino acid synthetic pathways are absent in Fusobacteria, they rely on their ability to import and

Fusobacterium nucleatum ATCC-25586 (IG)

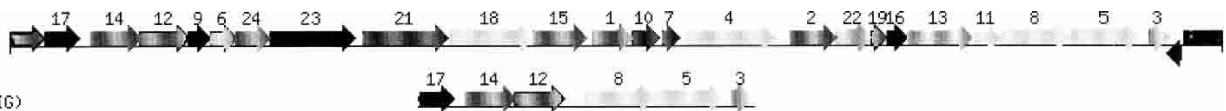

Fusobacter ium nucleatum vincentii ATCC-49256 (IG)

Figure 3 Deletion of the ethanolamine utilization operon in F. nucleatum sub spp vincentii. Between the ORFs for the dihydropteroate synthase (ORF12) and phosphoserine phosphatase (ORF 8) is the operon with 17 ORFs that encode enzymes for ethanolamine utilization found in $F$. nucleatum (Kapatral et al. 2002). The ORFs and their functional description are as follows: (17) GTP cyclohydrolase; (14) 2-Amino-4-hydroxy-6hydroxymethyl dihydropteridine pyrophosphokinase/dihydroneopterin aldolase (fusion protein); (12) dihydropteroate synthase; (9) EutS; (6) EutP; (24) two-component response regulator; (23) sensor kinase; (21) EutA; (18) ethanolamine ammonia-lyase heavy chain; (15) ethanolamine ammonia-lyase light chain; (1) EutL; (10) EutM (potential frame shift); (7) EutM (potential frame shift); (4) EutE; (2) cobalamin adenosyltransferase; (22) hypothetical protein; (19) EutN; (16) hypothetical protein; (13) ethanolamine permease; (11) EutQ; (8) phosphoserine phosphatase; (5) butanol dehydrogenase; (3) thioredoxin.

\section{Genome Research}




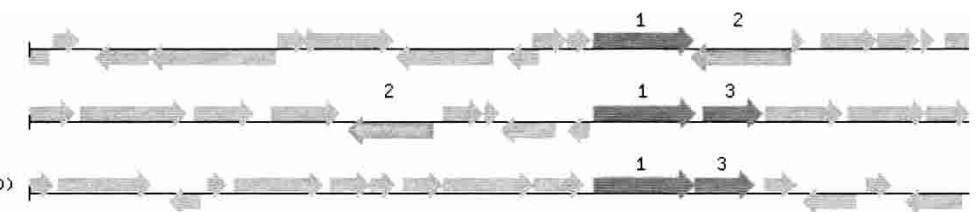

Figure 4 Organization of the ORFs that code for malolactic permease (1) and malolactic enzyme (3) in F. nucleatum sub spp vincentii (FNV). These pairs of occurrences are also found in Streptococcus mutans (SM) and Mycobacterium tuberculosis (MT). These ORFs are absent from F. nucleatum (FN).

degrade di- or oligo-peptides. Among the carboxypeptidases, two ORFs for Xaa-His dipeptidase have been identified in FNV, whereas FN has four such ORFs. ORFs for acylaminoacyl, pyroglutamyl peptidase-I, leucyl, aspartyl, prolyl, membrane alanine-peptidase, XAA-pro, T family peptidases, Xaa-pro dipeptidase, and serine-type D-ala-ala-carboxypeptidases have been identified in both genomes. In addition, ORFs for a membrane-associated peptidase, zinc metalloprotease, Osialoglycoprotein endopeptidases, and oligoendopeptidase $\mathrm{F}$ family have been found in both genomes (Table 6).

\section{ABC Transporters}

There are major differences in the peptide and iron $\mathrm{ABC}$ transporters between the two Fusobacterium sub spp. Proteins are degraded and taken in either as di-peptides or oligo-peptides via the peptide transporters (Table 7). Transporters for phosphonates, nickel, and iron are found in both genomes. In addition, $\mathrm{FN}$ has three iron-specific $\mathrm{ABC}$ transporters, of which two are arranged in tandem. The third iron-specific transporter is located adjacent to the outer membrane TonBdependent receptor. Three copies of the putative hemin outer

Table 6. Occurrence and Types of Peptidases in F. nucleatum sub spp

\begin{tabular}{l} 
Peptidase \\
\hline 1. Aminoacyl-histidine dipeptidase \\
2. Aspartyl aminopeptidase \\
3. Cell wall endopeptidase family $\mathrm{m} 23 / \mathrm{m} 37$ \\
4. Cytosol aminopeptidase \\
5. D-alanyl-D-alanine carboxypeptidase \\
6. Deblocking aminopeptidase \\
7. Gamma-glutamyltranspeptidase \\
8. Lipoprotein signal peptidase \\
9. Metalloendopeptidases \\
10. Methionine aminopeptidase \\
11. Multimodular transpeptidase E-transglycosylase \\
Pbp $1 \mathrm{~A}$
\end{tabular}

12. Multimodular transpeptidase E-transglycosylase Pbp 1C

13. Oligoendopeptidase $F$

14. O-sialoglycoprotein endopeptidase

15. Peptidase

16. Peptidase $\mathrm{E}$

17. Peptidase $\mathrm{T}$

18. Proline iminopeptidase

19. Pyrrolidone-carboxylate peptidase

20. Signal peptidase I

21. Thermostable carboxypeptidase 1

22. Xaa-His dipeptidase

23. Xaa-Pro aminopeptidase

24. Xaa-Pro dipeptidase

FN FNV

Plus sign indicates presence of the peptidase.

Minus sign indicates absence of the peptidase. membrane receptors are found in FNV, whereas five ORFs in FN have been identified. FNV also contains an operon for ferrous iron transporter FeoAB (FNV00510, FNV00511, FNV00512); these transporters are absent from FN. FeoB is commonly found in anaerobes, because under anaerobic conditions, soluble iron is present in the ferrous form, and is therefore more accessible than ferric iron for transport. A gene (FNV00582) with sequence similarity to an unknown type ABC transporter of T. denticola has been found in FNV. Another ORF encoding an ATP-binding protein along with a transporter of unknown function has been identified only in FNV. However, ORFs for glutaminase, clustered with a sodium/amino acid symporter, phospholipid, and LPS-flipping protein MsbA, are found in both genomes.

\section{Phosphotransferases}

Both FN and FNV have an identical sugar phosphotransferase (PTS) system. For example, in FNV genes, encoding EI and one copy of HPr are in an operon, whereas the second Hpr copy lies elsewhere on the chromosome. Both subspecies that have a PTS system for fructose have fused IIA, IIB, and IIC subunits and a second system similar to the glucose and $\mathrm{N}$ acetylglucosamine BC subunits. In addition, there are two IIA subunits, one of which likely interacts with the BC subunit. The P-type ATPases for export of zinc, copper, calcium, and cadmium have been identified in FN and FNV. Both FN and FNV have two copies of arsenate export membrane proteins that could function alone or with their cognate ATPases.

\section{Lipid Biosynthesis}

All of the ORFs in FNV for lipid, phospholipid synthesis, and LPS are similar to those in F. nucleatum (Kapatral et al. 2002). FN has two licABC operons, whereas one in the sequenced region of FNV has been identified. There is an orphan lic C and licA ORF distant from the licABC operon. The licD ORF is displaced in both the Fusobacterium sub spp. unlike Haemophilus influenzae (Kapatral et al. 2002). The fatty acid biosynthesis genes such as oxoacyl synthase, oxoacyl reductase, and enoyl reductase, except for 3-hydroxyfecanoyl dehydratase, are identical in both genomes. Four ORFs for oxoacyl synthase in FNV and three in FN have been identified.

\section{Bioenergetics}

Fusobacteria generate most of its energy equivalents by fermentation of amino acids and carbohydrates. The primary respiratory system for Fusobacterium sub spp. is oxidative phosphorylation. Fusobacterium sub spp. lacks classical components of the aerobic electron transfer chain such as complex I (NADH-quinone oxidoreductase, (EC 1.6.5.3), complex II (succinate:quinone oxidoreductase, EC 1.3.9.1), complex III (ubiquinone-cytochrome c reductase, EC 1.10.2.2), terminal, 
Table 7. Peptide ABC Transporter Family Proteins of $F$. nucleatum sub spp.

\begin{tabular}{llcc}
\hline Transport type & \multicolumn{1}{c}{ Subunit } & FN ORF \# & FNV ORF \# \\
\hline 1 Dipeptide & Binding protein & 191 & $2162 / 2163$ \\
& Membrane protein & 189,188 & 2164,2165 \\
& ATPases & 187,186 & 2166,2167 \\
2 Dipeptide & Binding protein & 2806 & $180 / 181 / 182$ \\
& Membrane protein & 1253,1254 & 529,528 \\
& ATPases & 2621 & 527 \\
& & $(2$ domains) & $(2$ domains) \\
& Binding protein & 1351 & 1261 \\
Dipeptide & Membrane protein & 1352,1353 & 1262,1264 \\
& ATPases & 2283,1354 & 1265,1267 \\
4 Dipeptide & Binding protein & 2842 & - \\
& Membrane protein & 1792,1045 & - \\
5 Oligopeptide & ATPases & 780,781 & 687 \\
& Binding protein & 1561 & 688,689 \\
& Membrane protein & 2952,619 & $690 / 691,-$ \\
6 Oligopeptide & ATPases & 618,617 & 2183 \\
& Binding protein & 770 & $2180,2181 / 2182$ \\
& Membrane protein & 772,771 & $2184-2185,2186$ \\
7 Oligopeptide & ATPases & 769,768 & 767 \\
& Binding protein & - & 768,769 \\
& Membrane protein & - & $770-771,701$ \\
8 Dipeptide & ATPases & - & 598 \\
9 Oligopeptide & Binding protein & 848 & 937 \\
\hline
\end{tabular}

ORF numbers separated by a slash (/) represent a frameshift or split ORFs.

Minus sign indicates absence of the transporter.

ORF numbers separated by a comma (,) represent the presence of more than one full protein.

Pseudomonas aeruginosa. ORFs for $\mathrm{Na}^{+}$-translocating NADH-quinone dehydrogenase, $\mathrm{Na}^{+}$transporting ATP synthase, and a number of $\mathrm{Na}^{+} / \mathrm{H}^{+}$transporters are present in both species. Both $\mathrm{Na}^{+} / \mathrm{H}^{+}$antiporters and $\mathrm{H}^{+}$-translocating ATP syntheses serve as major contributors to the proton-based electrochemical gradient. On the basis of this analysis, we conclude that Fusobacteria has a limited capability for aerobic respiration and is suited for an anaerobic life style, consistent with its growth in vitro and in anaerobic pockets of mouth (Dzink et al. 1990).

\section{Cl-Carbon Metabolism}

ORFs for enzymes associated with one carbon metabolism are not localized identically in the two genomes. For example, the region downstream of the ORF for formate-tetrahydrofolate ligase (FNV01815) in FN contains part of an ORF for an ABC transporter, however, in FNV there are ORFs for two transposases and a cobyric acid synthase. The ORF for cobyric acid synthase is not close to the formatetetrahydrofolate ligase gene in FN.

and complex IV (cytochrome or quinol reductase). Nevertheless, they can survive under microaerophilic conditions because of the presence of NADH oxidase (EC 1.6.99.3).

The anaerobic respiration system in Fusobacteria is complex. Several major routes for electrons that enter into the respiratory chain have been identified, including $\mathrm{Na}^{+}$translocating NADH-quinone dehydrogenase (EC 1.6.5.-), NAD (P) $\mathrm{H}^{+}$dehydrogenase (EC 1.6.99.-), electron transfer flavoprotein-ubiquinone oxidoreductase system (EC 1.5.5.1), and D- and L-lactate dehydrogenase (EC 1.1.1.28 and EC 1.1.1.27). All of the enzyme complexes for terminal electron acceptors, including those that oxidize quinones, are absent, and only ORFs for mercuric reductase and the flavoprotein subunit of fumarate reductase are found. The presence of the latter ORF may not be functional because iron-sulfur and hydrophobic anchor subunits are absent. In addition to the above terminal electron acceptors, only FNV has the entire operon (three ORFs) for anaerobic sulfite reductase. Sulfite reductase would allow FNV to generate sulfide from sulfite. The subunit B of sulfite reductase has an FAD-binding motif, a large hydrophobic domain, and could interact with a cytosolic ferredoxin to accept electrons from the quinone pool. ORFs for ubiquinone synthesis have not been identified. There are two ORFs that encode enzymes (O-succinylbenzoate CoA-synthase and 1,4dihydroxy-2-naphthoate octaprenyl transferase) involved in menaquinone biosynthesis in FN, but none in FNV. An ORF for 1,4-dihydroxy-2-naphthoate octaprenyltransferase is found in FNV. It is not surprising to find menaquinone as an electron transport component in Fusobacteria considering its anaerobic lifestyle. Cytochrome-based electron transfer reactions are absent. Fusobacteria have a $\mathrm{Na}^{+}$exchange-based and proton-based cycle to generate a transmembrane electrochemical gradient similar to those found in Vibrio cholerae and
Both FN and FNV contain ORFs for an efflux pump and the methyltransferase in the same orientation, but the methyltransferase is only about one-fourth the size of the $E$. coli ortholog (Old et al. 1990). In both genomes, there are two ORFs for thymidylate synthase with the flanking genes rearranged. Comparing a 10-kb sequence upstream of the ORF FNV02128, we identified two FNV ORFs with unknown function (FNV02123, FNV02124), followed by an ORF for the MarR family of transcriptional regulators. However, the flanking ORFs are different in $\mathrm{FN}$, and the MarR transcriptional regulator (FN00689) is located elsewhere in the genome. The second ORF for thymidylate synthase has identical downstream regions in both genomes, but the two ORFs are absent from the upstream region in FN (Fig. 5).

\section{Secretion and Outer Membrane Proteins}

In general, Fusobacteria are not known to secrete proteins into the external medium, which correlates with the lack of genes encoding components of secretion systems (Kapatral et al. 2002). Genes for the type-I secretion (RND family) of acriflavin resistance is present in both genomes. However, only four ORFs of the general secretion pathway (type II) protein D, E, $\mathrm{F}$, and $\mathrm{G}$ have been identified in both FN and FNV, indicating the loss of the GSP secretion system. Sec-dependent secretion involving genes for protein translocation ( $\sec A, \sec D$, secE, $\sec F$, sec $Y$, and trigger factor) and a protein translocase system are present in FNV. Two copies of the protein translocase secG have been identified in FNV, one of which has a frame shift. Seven Fusobacterium outer membrane (FOM) proteins ( $>200$ $\mathrm{kD}$ ) similar to the nine FOMs in FN (Kapatral et al. 2002) have been identified in FNV. Six outer membrane proteins, two TolC proteins, and one $\mathrm{P} 1$ protein precursor protein are found in both subspecies. 


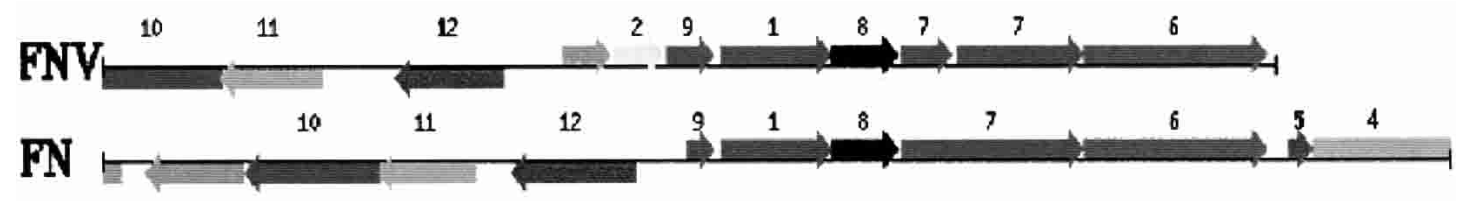

Figure 5 Gene shuffling flanking a thymidylate synthase locus. The ORFs represented among FN and FNV genomes are colored and numbered as a set according to homology and paired bidirectional best hits. The sets are (1) thymidylate synthase, (2) hypothetical protein, (3) hypothetical protein, (4) copper-exporting ATPase, (5) COP associated protein, (6) poly(A) polymerase/tRNA nucleotidyltransferase, (7) potassium uptake protein, (8) dihydrofolate reductase, (9) hypothetical protein, (10) thiamine-binding protein, (11) thiamine transport system, and (12) hypothetical protein. Set 1 contains ORFs FNV01239 and FN01321.

\section{Virulence and Antibiotic Resistance}

Both Fusobacterium sub spp. have genes for hemolysin, hemolysin precursor, and hemolysin III. Two copies of the paired hemolysin activator precursor and hemolysin are found in the sequenced region of FNV, whereas FN has three copies. An ORF in FNV with sequence similarity to the RhuM protein is found in the pathogenicity island (SPI-3) of Salmonella typhimurium. The ORF for neutrophil-activating protein and ABC transporter protein is found in FN, but not in FNV. Compared with FN, the operon for lipooligosaccharide (LOS) biosynthesis is shuffled in FNV. Downstream from the LOS choline transferase, there are ORFs for a hydrogenase, mannose-1phosphate guanyltransferase, and serine threonine phosphatase. A single ORF for S-layer protein similar to that found in $T$. denticola, C. botulinum, and $P$. gingivalis.

Genes that confer resistance to acriflavin, florfenicol (chloramphenicol) (Schwarz et al. 2000), multi-drug, daunorubicin, bacitracin, and nitroimidazole are found in both genomes. Only FN has the ORFs for a multidrug resistance protein $\mathrm{ABC}$ transporter, $\beta$-lactamase protein, and bleomycin resistance proteins.

\section{Signal Transduction and Stress}

Singal transduction systems in Fusobacteria are not known, From genome sequence, we have identified a two-component signaling system. A single pair of the two-component response regulator and its cognate kinase of the Gram-positive YesN-YesM family, autolysin family (LytS-LytR) have been identified in FNV similar to one found in FN (Kapatral et al. 2002). A third pair of two-component signal transduction system proteins similar to CzcR-CzcS found in Bacillus spp. has been identified in both the Fusobacterium sub spp. In addition, there is a single ORF in FNV for a histidine kinase with similarity to the sporulation kinase $\mathrm{B}$ of $\mathrm{B}$. cereus.

An ORF with similarity to a serine/threonine phosphatase has been identified downstream of the lipooligosaccharide operon in FNV. In addition, there are two genes (FNV01726 and FNV00759) for eukaryotic-type serine/ threonine kinase, one of which (FNV01726) is similar to one found in Staphylococcus aureus. The second kinase (FNV00759) has sequence similarity to the one in FN and Thermonospora fusca. The roles of these kinases in Fusobacteria are unknown.

Fusobacteria can tolerate heat, but not cold stress. Heatshock stress proteins such as the heat-inducible transcription factor HrcA, GrpE, DnaK, DnaJ, and chaperone protein HtpG have been found in both FN and FNV genomes. Genes for catalase-peroxidase enzymes that are involved in oxidative stress are absent in sequenced regions of both FNV and FN, but thioredoxin/glutaredoxin enzymes could be used to scavenge peroxide radicals. A gene for the carbon starvation protein A has been identified in both species.
Although FNV does not have complete DNA uptake competence systems, two ORFs with sequence similarities to the ComE and ComF proteins of $B$. fragilis have been identified.

\section{Phages and Transposons}

Cryptic phages have been identified in FNV; six phage contigs encoding 110 ORFs have been found. The average GC content of the phage DNA is about $28 \%$, and codon usage is similar to the chromosome DNA. Neither the origin nor occurrence of phages has been reported in this genus. One of the phage regions contains 66 ORFs and two have 14 ORFs, each with amino acid sequence similarity to the phage-like element PBSX protein XkdK, XkdM, XdkT, etc. of Desulfitobacterium halfniense DCB-2 phage. The fourth region has six ORFs with amino acid sequences similar to those of the Gram-negative bacteriophage P2. The phage regions 5 and 6 have three and seven phage ORFs, respectively, with sequences similar to the Gram-positive bacteriophage TP901. There are no phage sequences in $\mathrm{FN}$.

Segments of DNA are often lost or displaced during transposition, excision, or recombination events. An example of such a case is the ORF for glycerophosphoryl diester phosphodiesterase (FNV02349) and a $\mathrm{Na}^{+}$-inked D-alanine glycine permease (FNV02348), which are complete in the FNV genome but truncated in FN (Fig. 6). Downstream of permease, is a gene similar to the ATPase necessary for chromosome architecture/replication, and a gene for serine protease. Similarly, an ORF for sodium-coupled amino acid transporter is present in FNV, whereas this transporter gene is truncated in FN. There are 15 ORFs with sequence similarity to transposases. Four ORFs have frame shifts and have resulted in 19 ORFs with transposase assignments. Most of the transposases are truncated and do not have IS elements or terminal repeats, and are perhaps nonfunctional.

\section{Conclusion}

Draft genome with a good coverage provides sufficient information to reconstruct core metabolism of a given genome (Selkov et al. 2000). However, the presence of a complete genome sequence of a very closely related species provides a benchmark for comparisons and identification of unique features present in both genomes, as in Salmonella spp. (Edwards et al. 2002), Xylella spp. (Bhattacharyya et al. 2002), and Yersinia pestis (Deng et al. 2002). We have previously sequenced and analyzed $F$. nucleatum strain ATCC 25586 (Kapatral et al. 2002). In this work, we have presented the draft sequence and analysis of the poorly studied $F$. nucleatum sub $s p p$ vincentii. Using the sequence information for $\mathrm{FN}$ as benchmark, the comparative genome analysis of the FNV genome was performed using the ERGO genome bioinformatics suite. Although the metabolic capabilities of these two genomes are 


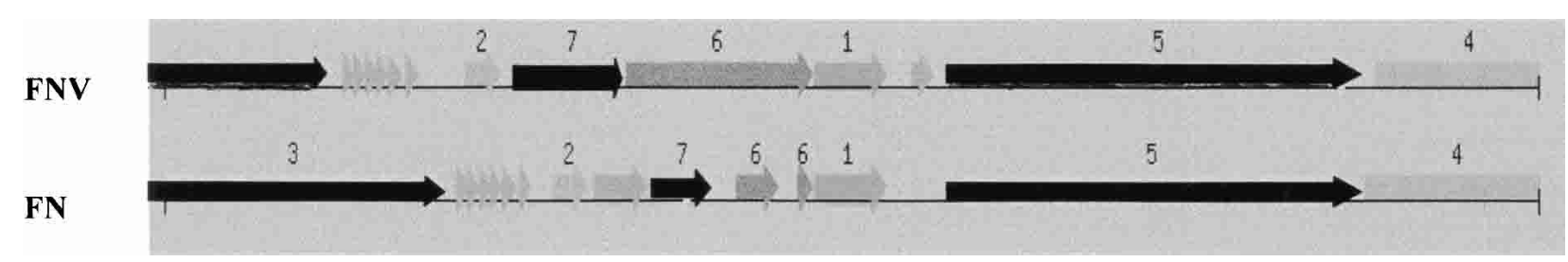

Figure 6 Contig display of the two Fusobacterium sub spp. genomes showing gain of functions in FNV. Similarly colored ORFs (arrows) indicate the presence of similar genes. (1) ATPase associated with chromosome architecture; (2) hypothetical protein; (3) O-linked GLCNAC transferase; (4) Xaa-Pro peptidase; (5) serine protease; (6) Na+-linked D-alanine glycine permease (truncated in FN); (7) glycerophosphoryl diester phosphodiesterase; (8) N-linked alanine-glycine permease (truncated in FN). The numbering is based on the bidirectional best hit.

similar, there are 441 ORFs in FNV, ORFs that are not present in FN. Some of these include malolactic permease, glutamine synthetase, and glycosyltransferases. The sugar component of the O-antigen in FNV LPS might have galactopyranose, galactoronate, and sialic acid. Two ABC transporters, anaerobic ferrous transporter operon, and anaerobic sulfite reductase have also been identified in FNV.

In general, several operons in FNV are rearranged compared with $\mathrm{FN}$, in spite of a remarkable synteny over $85 \%$ of the genome. The presence of unique peptidases for protein degradation broadens the substrate range for growth in FNV. Four types of restriction-modification systems (type I, type II, type III, and mrr) are found in FNV that restricts foreign DNA. Thus, in addition to amino acids and carbohydrates, ribose nucleotides could also serve as a source for carbon and nitrogen. FNV appears to have lost several genes and, therefore, is limited for survival under certain conditions, including ethanolamine utilization, thermostable carboxypeptidase, $\gamma$ glutamyl-transpeptidase, and deblocking aminopeptidases. Occurrence of eukaryotic-type serine/threonine kinase and phosphatase suggests unique signal transduction pathways that might be present in FNV. Occurrence of genes for resistance to acriflavin, bacitracin, bleomycin, daunorubicin, and florfenicol allow survival of these bacteria during antibiotic treatment.

\section{METHODS}

\section{DNA Sequence and Analysis}

The strain F. nucleatum sub spp vincentii ATCC 49256 was purchased from the American Type Culture Collection. A plasmid genomic library in pGEM3 (Promega) was constructed in $E$. coli $\mathrm{DH} 5 \alpha$ and shotgun sequenced, as described for $F$. nucleatum ATCC 25586 (Kapatral et al. 2002). A total of 13,000 plasmid templates, about 20,000 sequencing reactions, were carried out using Applied Biosystems 3700 DNA sequencers. The DNA sequences were assembled using PHRED-PHRAP, and edited manually using CONSED to an average coverage of 6.4-fold. The total number of DNA base pairs for the complete genome FN is 2,174,500 (Kapatral et al. 2002) and for FNV is $2,118,259$, covering $98 \%$ of the FN genome. The 2,118,259-bp DNA sequence was assembled into 302 contigs, with the smallest being $334 \mathrm{bp}$ and the largest being 61,432 bp. An ORF-calling program developed at Integrated Genomics Inc. was used to identify putative ORFs. The genome analysis was performed using the Integrated Genomics ERGO bioinformatics suite, containing $>500$ genomes covering the three domains of life, of which $>220$ genomes are completely or almost completely sequenced. The annotations of the ORFs were carried out using the Integrated Genomics ERGO threestep procedure as described for the strain F. nucleatum ATCC 25586 (Kapatral et al. 2002) and Brucella melitensis (Del Vecchio et al. 2002).
Comparative genome analysis technology such as GenomeWalk and Workbench (both available through the ERGO suite) were used. GenomeWalk provides a graphical representation of the whole-genome comparison that facilitates the identification of unique chromosomal regions between related genomes. Workbench allows the identification of the common and unique clusters of genes between genomes (Bhattacharyya et al. 2002). Protein family clusters between the two Fusobacterium sub spp. genomes were computed with a BLAST cutoff score of $\mathrm{E}^{-10}$. Chromosomal clusters (potential operons) were identified as described earlier (Overbeek et al. 1999; Bhattacharyya et al. 2002).

\section{ACKNOWLEDGMENTS}

This work was supported by NIH grant R4GM61431-02. We thank the Integrated Genomics sequencing and assembly team for their contribution to the genome project.

The publication costs of this article were defrayed in part by payment of page charges. This article must therefore be hereby marked "advertisement" in accordance with 18 USC section 1734 solely to indicate this fact.

\section{REFERENCES}

Baker, J.J., van der Drift, C., and Stadtman, T.C. 1973. Purification and properties of $\beta$-lysine mutase, a pyridoxal phosphate and B12 coenzyme dependent enzyme. Biochemistry 12: 1054-1063.

Bhattacharyya, A., Stilwagen, S., Lykidis, A., Anderson, I., Bernal, A. D'Souza, M., Ivanova, N., Kapatral, V., Larsen, N., Los, T., et al. 2002. Whole-genome comparative analysis of three phytopathogenic Xylella fastidiosa strains. Proc. Natl. Acad. Sci. 19: $12403-12408$.

Belanger, M., Burrows, L.L., and Lam, J.S. 1999. Functional analysis of genes responsible for the synthesis of the B-band $\mathrm{O}$ antigen of Pseudomonas aeruginosa serotype O6 lipopolysaccharide. Microbiology 145: 3505-3521.

Bolstad, A.I. and Jensen, H.B. 1993. Methlyation of adenine and cytosine in some strains of Fusobacterium nucleatum. Microb. Pathog. 14: 117-121.

Bolstad, A.I., Jensen, H.B., and Bakken, V. 1996. Taxonomy, biology, and periodontal aspects of Fusobacterium nucleatum. Clin. Microbiol. Rev. 9: 55-71.

Carlier, J.P., Henry, C., Lorin, V., and Rouffignat, K. 1997. Conversion of DL-threonine, D-threonine and 2-oxobutyrate into propionate and 2-hydroxybutyrate by Fusobacterium species. Lett. Appl. Microbiol. 25: 371-374.

Chang, C.H. and Frey, P.A. 2000. Cloning, sequencing, heterologous expression, purification and characterization of adenosylcobalamin-dependent D-lysine 5,6-aminomutase from Clostridium sticklandii. J. Biol. Chem. 275: 106-114.

Creuzenet, C., Schur, M.J., Li, J., Wakarchuk, W.W., and Lam, J. 2000. FlaA1: A new bifunctional UDP-GlcNAc C6 dehydratase/C4 reductase from Helicobacter pylori. J. Biol. Chem. 275: $34873-34880$

DelVecchio, V.G., Kapatral, V., Redkar, R.J., Patra, G., Mujer, C., Los, T., Ivanova, N., Anderson, I., Bhattacharyya, A., Lykidis, A., et al. 2002. The genome sequence of the facultative intracellular pathogen Brucella melitensis. Proc. Natl. Acad. Sci. 99: 443-448.

\section{Genome Research


Deng, W., Burland, B., Plunkett III, G., Boutin, A., Mahew, G.F., Liss, P., Perna, N.T., Rose, D.J., Mau, B., Zhou, S., et al. 2002. Genome sequence of Yersinia pestis KIM. J. Bacteriol. 16: 4601-4611.

Dzink, J.L., Sheenan, M.T., and Socransky, S.S. 1990. Proposal of three subspecies of Fusobacterium nucleatum Knorr 1922: Fusobacterium nucleatum subsp. nucleatum subsp. nov., comb. nov.; Fusobacterium nucleatum subsp. polymorphum subsp. nov., nom. rev., comb. nov.; and Fusobacterium nucleatum subsp. vincentii subsp. nov., nom. rev., comb. nov. Int. J. Syst. Bacteriol. 40: $74-78$.

Edwards, R.A., Olsen, G.J., and Maloy, S.R. 2002. Comparative genomics of closely related Salmonellae. Trends Microbiol. 10: $94-99$.

Gharbia, S.E. and Shah, H.N. 1988. Characteristics of glutamate dehydrogenase, a new diagnostic marker for the genus Fusobacterium J. Gen. Microbiol. 134: 327-332.

. 1990. Heterogeneity within Fusobacterium nucleatum, proposal of four subspecies. Lett. Appl. Microbiol. 10: 105-108. . 1992. Fusobacterium nucleatum subsp. fusiforme subsp. nov. and Fusobacterium nucleatum subsp. animalis subsp. nov. as additional subspecies within Fusobacterium nucleatum. Int. J. Syst. Bacteriol. 42: 296-298.

Han, Y.W., Shi, W., Huang, G.T., Haake, S.K., Park, N.H., Kuramitsu, H., and Genco, R.J. 2000. Interactions between periodontal bacteria and human oral epithelial cells: Fusobacterium nucleatum adheres to and invades epithelial cells. Infect and Immun. 68: $3140-3146$.

Hermansson, K., Perry, M.B., Altman, E., Brisson, J.R., and Garcia, M.M. 1993. Structural studies of the O-antigenic polysaccharide of Fusobacterium necrophorum. Eur. J. Biochem. 212: 801-809.

Kapatral, V., Anderson, I., Ivanova, N., Reznik, G., Los, T., Lykidis, A., Bhattacharyya, A., Bartman, A., Gardner, W., Grechkin, G., et al. 2002. Genome sequence and analysis of the oral bacterium Fusobacterium nucleatum strain ATCC 25586. J. Bacteriol.

184: $2005-2018$.

Kolenbrander, P.E., Andersen, R.N., Blehert, D.S., England, P.G,
Foster, J.S., and Palmer Jr., R.J. 2002. Communication among oral bacteria. Microbiol. Mol. Biol. Rev. 66: 486-505.

Krishnaprakornkit, S., Kimball, J.R., Weinberg, A., Darveau, R.P. Bainbridge, B.W., and Dale B.A. 2000. Inducible expression of human $\beta$ defensin by Fusobacterium nucleatum in oral epithelial cells: Multiple signaling pathways and role of commensal bacteria in innate immunity and the epithelial barrier. Infect. Immun. 68: 2907-2915.

Moore, W.E.C. and Moore, L.V.H. 1994. The bacteria of periodontal diseases. Periodontology 5: 66-77.

Old, I.G., Margarita, D., Glass, R.E., and Girons, I. 1990. Nucleotide sequence of the metH gene of Escherichia coli K-12 and comparison with that of Salmonella typhimurium LT2. Gene 87: 15-21.

Onoue, S., Niwa, M., Isshiki, Y., and Kawahara, K. 1996. Extraction and characterization of the smooth-type lipopolysaccharide from Fusobacterium nucleatum JCM 8532 and its biological activities. Microbiol. Immunol. 40: 323-333.

Overbeek, R., Fonstein, M., D'Souza, M., Pusch, G.D., and Maltsev, N. 1999. The use of gene clusters to infer functional coupling. Proc. Natl. Acad. Sci. 96: 2896-2901.

Schwarz, S., Werckenthin, C., and Kehrenberg, C. 2000. Identification of a plasmid borne chloramphenicol-florfenicol resistance gene in Staphylococcus sciuri. Anti. Microbial. Chem. 44: $2530-2533$

Selkov, E., Overbeek, R., Kogan, Y., Chu, L., Vonstein, V., Holmes D., Silver, S., Haselkorn, R., and Fonstein, M. 2000. Functional analysis of gapped microbial genomes: Amino acid metabolism of Thiobacillus ferrooxidans. Proc. Natl. Acad. Sci. 97: 35093514.

Wang, L., Curd, H., Qu, W., and Reeves, P.R. 1998. Sequencing of Escherichia coli $\mathrm{O} 111 \mathrm{O}$-antigen gene cluster and identification of O111-specific genes. J. Clin. Microbiol. 36: 3182-3187.

Received June 28, 2002; accepted in revised form February 25, 2003.
Genome Research www.genome.org 


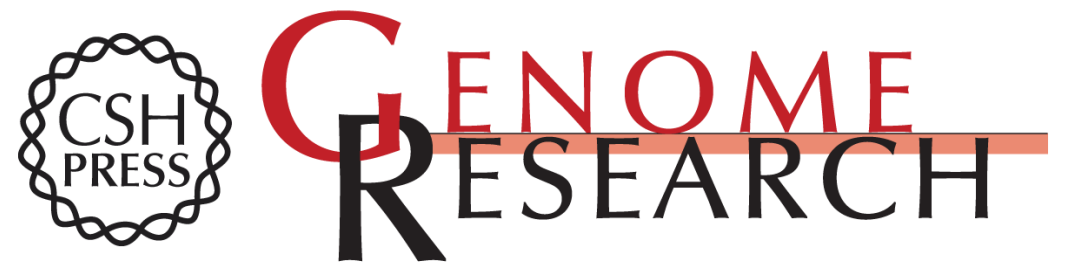

\section{Genome Analysis of $F$. nucleatum sub spp vincentii and Its Comparison With the Genome of F. nucleatum ATCC 25586}

Vinayak Kapatral, Natalia Ivanova, lain Anderson, et al.

Genome Res. 2003 13: 1180-1189

Access the most recent version at doi:10.1101/gr.566003

References This article cites 24 articles, 11 of which can be accessed free at:

http://genome.cshlp.org/content/13/6a/1180.full.html\#ref-list-1

\section{License}

Email Alerting Receive free email alerts when new articles cite this article - sign up in the box at the Service top right corner of the article or click here.

\section{Affordable, Accurate Sequencing.}

